Canadian University Music Review

Revue de musique des universités canadiennes

\title{
Situation de la synthèse sonore par ordinateur
}

\section{Denis Lorrain}

Numéro 3, 1982

URI : https://id.erudit.org/iderudit/1013833ar

DOI : https://doi.org/10.7202/1013833ar

Aller au sommaire du numéro

Éditeur(s)

Canadian University Music Society / Société de musique des universités

canadiennes

ISSN

0710-0353 (imprimé)

2291-2436 (numérique)

Découvrir la revue

Citer cet article

Lorrain, D. (1982). Situation de la synthèse sonore par ordinateur. Canadian University Music Review / Revue de musique des universités canadiennes, (3),

170-202. https://doi.org/10.7202/1013833ar

All Rights Reserved (C Canadian University Music Society / Société de musique des universités canadiennes, 1982
Ce document est protégé par la loi sur le droit d'auteur. L'utilisation des services d'Érudit (y compris la reproduction) est assujettie à sa politique d'utilisation que vous pouvez consulter en ligne.

https://apropos.erudit.org/fr/usagers/politique-dutilisation/ 


\title{
SITUATION DE LA SYNTHÈSE SONORE PAR ORDINATEUR
}

\author{
Denis Lorrain
}

Nous allons faire ici un rapide survol du domaine de la synthèse numérique, non pas tellement d'un point de vue scientifique et technologique, ${ }^{1}$ mais plutôt de celui, parmi 'ses utilisateurs, des compositeurs - dont on peut présumer qu'ils forment une certaine catégorie d'honnêtes hommes, bien qu'ils puissent à l'occasion entreprendre des démarches non prévues par les techniciens. Quelle est donc cette méthode de production de sons, selon quelles approches peut-elle être abordée, et quelles en sont les incidences musicales?

Sur la base de quelques notions simples, qui nous serviront à décrire brièvement les rudiments théoriques de la synthèse sonore numérique et les conditions pratiques de son utilisation, se dégageront en effet des implications musicales importantes, bien qu'elles ne soient pas aussi radicales que ce que pourraient laisser croire à première vue les mirages technologiques qui entourent ce processus. Nous donnerons ensuite un aperçu des approches pratiquées dans ce domaine, basées sur des conceptions acoustiques et musicales fondamentales. Les divers retentissements de ces approches montreront que la synthèse sonore numérique procure essentiellement aux compositeurs l'annexion de nouveaux et très vastes domaines de liberté. Mais la liberté n'est pas chose facile ... ${ }^{2}$

\section{La synthèse sonore numérique : techniques et implications}

Tout phénomène sonore - dont ce qu'on appelle musique est un cas particulier - se manifeste par des variations de la pression atmosphérique (mouvement de va-et-vient des molécules gazeuses de l'air) qui atteignent le tympan, sont transmises à l'oreille interne qui en effectue une certaine analyse et achemine des

Revue de musique des universités canadiennes, $\mathrm{N}^{\circ} 3,1982$. 
impulsions nerveuses au cerveau. Non seulement le son se manifeste ainsi, mais il est cela : quand nous parlons de son, nous parlons de variations de pression perçues par une oreille. Ces variations sont causées par les vibrations d'un corps communiquées à l'air ambiant. Appelons ces variations de pression des ondes acoustiques.

Le haut-parleur est le corps vibratoire le plus général que nous possédions. Il suffit d'appliquer aux bornes de cet appareil les tensions électriques adéquates pour produire un type quelconque de variations de pression atmosphérique audibles; pour produire un son quelconque - qu'il s'agisse de la simulation ou de la reproduction des vibrations d'un corps matériel (violon, voix, machine, etc.) ou directement des variations de tension de circuits électroniques (oscillateurs, filtres, modulateurs, etc.)-, pourvu que la fonction de temps ainsi convertie en énergie vibratoire soit située dans l'aire audible, c'est-à-dire : 1) d'intensité suffisante mais, si possible, en deçà d'un seuil douloureux, et 2) de fréquence comprise entre $20 \mathrm{~Hz}$. (cycles par seconde) et $20000 \mathrm{~Hz}$. approximativement.

D'autre part, l'outil le plus général dont nous disposions pour effectuer des manipulations quelconques de ces fonctions de temps, est sans conteste l'ordinateur numérique - même s'il n'en est pas toujours et partout, à l'heure actuelle, le plus pratique ou le plus efficace : en effet, cette machine est neutre, en ce sens qu'elle est un puissant manipulateur (processor) d'informations, mais sans aucune spécialisation ; avec l'ordinateur, tout est, ou serait toujours possible.

Il s'élève cependant une frontière entre le domaine de l'ordinateur, qui est celui du traitement d'informations discrètes, et notre univers acoustique, qui est celui de variations continues de la pression atmosphérique. Le passage de cette frontière est assuré par un processus de conversion, soit analogique-numérique (du domaine acoustique vers l'ordinateur numérique), soit numériqueanalogique (en sens contraire : du discret vers le continu). Dans la perspective qui nous intéresse donc, celle de la synthèse sonore, un convertisseur numérique-analogique, suivi d'un filtre de lissage, permet de traduire, en variations continues de tension électrique, une liste de nombres discrets fournis par l'ordinateur. ${ }^{3}$ Lorsque ces nombres sont proportionnels aux amplitudes instantanées successives d'une onde acoustique quelconque, à des intervalles chronologiques connus, ils constituent un échantillonnage, c'est-à-dire une représentation symbolique (numérique) discrète 
des variations continues de l'onde acoustique à engendrer. La fonction continue obtenue à partir des échantillons peut ensuite être acheminée vers un haut-parleur et/ou un magnétophone.

Pour ce qui est de la fidélité acoustique de ce processus, de sa capacité de synthétiser des sons variés et de qualité suffisante, les deux dimensions de l'onde acoustique entrent en ligne de compte: celle des amplitudes et celle du temps (respectivement verticale et horizontale dans les représentations habituelles). Dans le sens du temps, il faut que l'échantillonnage soit assez fréquent pour rendre compte même des variations rapides de l'onde acoustique, c'est-à-dire même des fréquences élevées de l'air audible ; des débits suffisants, supérieurs à 20000 échantillons/seconde, sont aujourd'hui courants. ${ }^{4}$ Pour ce qui et de la résolution de l'échantillonnage dans le sens des variations fines de l'amplitude de l'onde acoustique, elle dépend du nombre de bits (unités d'information binaires) utilisés pour spécifier les amplitudes des échantillons: plus ce nombre est grand, plus nombreuses sont les amplitudes différentes susceptibles d'être représentées par les échantillons, plus fines les variations dont on peut rendre compte; des convertisseursadéquats sont disponibles pour ce faire. ${ }^{5}$

Pour la réalisation pratique de la synthèse numérique, deux contextes technologiques sont possibles, dont la différence apparaît primordialement dans leur efficacité temporelle. Un ordinateur d'usage général, simplement muni de convertisseurs numériqueanalogiques, ne possède pas à l'heure actuelle la rapidité nécessaire pour débiter à un taux adéquat les échantillons résultant des calculs préparatoires d'une sy nthèse de phénomènes acoustiques complexes : 6 il est nécessaire d'accumuler les échantillons sur une mémoire périphérique afin de les acheminer ultérieurement au convertisseur numérique-analogique, en temps différé, c'est-àdire après la fin du calcul de la totalité de la séquence sonore. Afin de remédier à cette situation, indésirable pour certains ou dans certaines circonstances, des périphériques spécialisés dans les opérations répétitives relativement simples, mais excessivement nombreuses, des calculs d'échantillons, et dotés d'une rapidité suffisante pour débiter en temps réel un taux d'échantillonnage acceptable, peuvent être adjoints à un ordinateur. Cette différence d'efficacité temporelle tient donc dans l'usage de dispositifs techniques différents, qui impliquent des conséquences profondes sur la généralité des systèmes de sythèse sonore et sur les approches qu'ils permettent.

Les systèmes en temps différé présentent le très grand avan- 
tage - préservé justement au prix du temps réel — d'être entièrement logiciels (software), c'est-à-dire composés uniquement de programmes exécutables sur tout ordinateur, et pouvant être à tout moment ajustés ou modifiés en fonction des besoins. D'autre part, la mise au point et l'expérimentation de méthodes entièrement nouvelles et imprévues de calcul d'ondes acoustiques peuvent être relativement aisément réalisées par programmation : c'est là l'essence même de ce qu'est un ordinateur. Enfin, il n'y a dans ce cadre technologique aucune limite à la complexité des calculs que l'utilisateur peut exiger - puisqu'il a déjà pris le parti d'accepter un délai appréciable entre la soumission d'une tâche à l'ordinateur et l'écoute de son résultat sonore. ${ }^{7}$ De toute façon le temps différé impose au compositeur une approche de la musique exclusivement par l'écriture, ${ }^{8}$ c'est-à-dire par la formulation rigoureuse d'une réflexion musicale préalable, certes nourrie d'expériences acquises et par des facultés d'imagination et d'audition intérieures, mais toujours chronologiquement dissociée du résultat sonore.

Les systèmes en temps réel, par contre, atteignent leur performance au prix de l'utilisation de matériels (hardware), c'est-àdire de circuits câblés dans une configuration définitive, dédiés à l'exécution rapide des calculs élémentaires de synthèse sonore proprement dite, sous les ordres d'un ordinateur gestionnaire. La modification d'un système matériel peut s'avérer difficile ou impossible, et il y a peu de chances qu'il soit utilisable pour la réalisation d'un processus de synthèse qui n'aurait pas été exactement prévu lors de sa construction. Il y a toujours, enfin, une limite matérielle aux capacités d'un tel système et à sa rapidité de calcul. Le temps réel offre cependant à l'utilisateur la possibilité d'une approche instrumentale de la synthèse sonore, en ce sens que la réponse acoustique immédiate du système permet l'interaction instantanée de l'audition avec des gestes accomplis sur différents dispositifs (touches, claviers, potentiomètres, etc.).

Plus précisément, deux types de périphériques en temps réel sont aujourd'hui utilisés. La majorité des synthétiseurs numériques accomplissent des opérations très spécialisées et sont conçus expressément pour certains processus bien définis de synthèse ; on peut dire, grosso modo, que ces périphériques réalisent la fonction d'un certain nombre d'oscillateurs capables d'engendrer des fonctions de temps spécifiées par l'utilisateur. ${ }^{9}$ Ces périphériques sont forcément limités aux objectifs pour lesquels ils ont été construits. Par contre, on a vu plus récemment le 
développement de processeurs sonores programmables (sound processors).$^{10}$ Ces périphériques sont des sous-ordinateurs relativement neutres, en ce sens qu'ils ne font qu'accomplir certaines opérations de base très élémentaires (additions, multiplications, consultations de tables, mémorisations d'échantillons, etc.), cependant avec une rapidité suffisante pour permettre le temps réel : par l'intermédiaire d'un ordinateur les commandant, ils peuvent être programmés en vue de réaliser divers types d'opérations spécialisées, et donc de processus de synthèse et/ou de traitement de signaux acoustiques. On retrouve donc dans ces appareils l'avantage essentiel des sytèmes logiciels : la programmabilité - mais encore avec la limite matérielle de leur capacité de traitement et de leur rapidité.

S'il est très intéressant, le développement spectaculaire des recherches dans ce domaine de la technologie de la synthèse sonore en temps réel occupe actuellement une place quelque peu exagérée parmi les applications musicales de l'informatique. ${ }^{11} \mathrm{Un}$ progrès technologique, en effet, n'apporte pas toujours en luimême la solution aux problèmes de son utilisation, de son intégration à un contexte plus vaste qu'il ne faut pas perdre de vue. La synthèse sonore numérique est maintenant une réalité - commerciale, même-, mais trop peu d'efforts sont déployés pour assurer sa fonction musicale :

Les véritables problèmes de l'informatique musicale ne sont pas dans le domaine de la synthèse sonore ... on accorde trop peu d'attention à la mise au point d'outils destinés à seconder le compositeur dans le contrôle ... des sons à l'intérieur d'un contexte musical (Buxton $1978: 31$ ). ${ }^{12}$

Et l'on peut ajouter : non seulement au développement d'outils, mais encore d'une pensée musicale qui devrait en même temps justifier ces outils et s'y adapter.

Par sa nature même, la synthèse sonore en temps différé implique un "grossissement de l'action de créer des sons, décomposée comme par un film au ralenti " (Chion 1976:3), ce qui rend possible chez le compositeur le mûrissement d'une conscience très aiguë de la réalisation acoustique fine des événements sonores qu'il met en jeu. Dans cette optique, la synthèse sonore s'inscrit tout à fait dans la lignée traditionnelle d'une composition réfléchie a priori, en ce sens qu'elle réalise acoustiquement une partition entièrement prédéterminée et méticuleusement rédigée 
par son auteur : il est vraisemblable que pour de nombreux compositeurs, le temps différé ne représente donc pas un handicap. Même en différé, la synthèse permet aussi d'outrepasser la dichotomie traditionnelle entre la composition et sa réalisation acoustique. Le compositeur, en effet, a le pouvoir de composer très précisément les sons mêmes qu'ilmet en action, et il est en tous cas responsable de leurs moindres caractéristiques. Il lui est alors possible d'adopter encore une attitude assez traditionnelle à cet égard, en utilisant la synthèse sonore comme un simple medium de réalisation acoustique - comme un instrument général et perfectionné. Dans ce cas sa démarche musicale s'avance jusqu'à la conception de l'agencement d'événements sonores autonomes, et à partir de ce point le relais est pris par une démarche acoustique : il y a juxtaposition, et complémentarité, de deux types de processus de natures essentiellement différentes. Il est cependant possible que le compositeur use d'une stratégie beaucoup plus fondamentale, et tente d'exploiter à fond le pouvoir essentiel de la synthèse sonore en temps différé, qui est de permettre la préparation entièrement libre d'ondes acoustiques : rien n'empêche alors de concevoir une extension de processus de type nettement compositionnel au-delà du niveau d'événements sonores destinés à être ensuite exécutés et réalisés par d'autres moyens, jusqu'à l'élaboration de l'onde acoustique elle-même, jusqu'au niveau ultime des échantillons sonores. Cette démarche englobante, de composition directe de l'onde sonore, échappe ainsi au recours exclusif à des modèles acoustiques dans les processus de synthèse.

Quoi qu'il en soit, le temps différé ne modifie donc pas considérablement la nature intrinsèque de la composition musicale ; il en provoque cependant un prolongement plus ou moins radical. À l'opposé, les systèmes en temps réel offrent la possibilité d'une approche typiquement instrumentale, allant éventuellement jusqu'à l'improvisation, le jeu en concert (live), etc. Dans ce cas, la synthèse numérique pourrait être ramenée à un simple progrès technologique sur les synthétiseurs analogiques aujourd'hui répandus, et ne concernant plus aussi directement la composition. Mais par contre, parce qu'elle bénéficie de l'environnement d'ordinateurs programmables, gérant les périphériques spécialisés dont nous avons parlé, la synthèse en temps réel donne naissance à des systèmes impliquant, à des degrés divers, l'informatisation de processus compositionnels ;13 en fait, les performances des périphériques en temps réel appellent nécessairement 
des organes de commande informatisés, puisqu'il serait sans doute absurde de sous-exploiter leurs capacités en les limitant, par exemple, à la dextérité pianistique de l'utilisateur s'ils n'étaient accessibles que par l'intermédiaire de claviers. La conjoncture est idéale, car l'ordinateur de commande se trouve justement libéré des tâches excessivement accaparantes du calcul des échantillons, et disponible pour l'accomplissement de fonctions d'un niveau plus élevé - musicales plutôt qu'acoustiques - qui, elles, sont à la portée de ses capacités de traitement en temps réel: le volume des données à traiter est énormément réduit - car il suffit d'adresser des commandes globales aux périphériques de synthèse-, de même que la cadence à fournir - car il ne s'agit plus que de gérer des événements musicaux, macroscopiques par rapport aux échantillons sonores.

L'environnement logiciel d'un système de synthèse en temps réel donne donc naissance à une notion assez nouvelle de création musicale assistée par ordinateur, englobant à la fois la composition proprement dite et sa réalisation acoustique immédiate, de manière que s'établit aisément un dialogue interactif entre l'ordinateur et le compositeur. ${ }^{14}$ Celui-ci peut alors écouter la réalisation acoustique d'ébauches ou de fragments plus ou moins complets de sa composition; en retour de ce feed-back il effectuera différentes modifications et adaptations, ou ira jusqu'à transformer radicalement son approche : il peut changer plus ou moins complètement les seuls paramètres de la réalisation acoustique sans altérer la séquence d'événements sonores, ou réviser plus ou moins profondément l'organisation compositionnelle du fragment en cours de mise au point, reprenser des agencements entre divers fragments, etc. ${ }^{15}$

La fonction du compositeur n'est pas fondamentalement modifiée par un tel environnment, en ce sens qu'il est toujours responsable de la production d'un objet fini, d'une oeuvre musicale qu'il considère comme achevée. Cependant son mode de travail se trouve considérablement altéré par la prise en charge par l'ordinateur ${ }^{16}$ d'une large part des tâches pratiques de l'écriture, de la manipulation des matériaux, etc., et par le fait que l'ordinateur peut se substituer à cette qualité essentielle du compositeur qui est l'audition intérieure incarnant une certaine imagination. On peut aller jusqu'à dire que la possibilité — ou la tentation - lui est offerte d'essayer avant de réfléchir, et même : plutôt que de réfléchir! D'autre part, il est inévitable que les prémisses musicales sur lesquelles est forcément basé un tel 
système, influencent certaines conceptions fondamentales de l'oeuvre. Il est en tous cas certain que de telles méthodes de composition déplacent l'investissement personnel représenté par l'élaboration de l'oeuvre, en allégeant considérablement tous ses aspects pratiques et, par le fait même, en accentuant l'importance des concepts abstraits qui sont à la source de la composition : puisque la réalisation concrète est, pour ainsi dire, assurée par l'ordinateur, le compositeur devient davantage concepteur, et beaucoup moins artisan - notre définition traditionnelle de l'artiste étant normalement située entre ces deux extrêmes. Comme dans les arts visuels, par exemple, l'ordinateur provoque ainsi un certain bouleversement des notions rattachées à l'art et à l'oeuvre. ${ }^{17}$

Mais puisqu'ils vont jusqu'à permettre l'improvisation, les systèmes de gestion de périphériques de synthèse sonore en temps réel peuvent ammener des bouleversements beaucoup plus radicaux. En effet, des processus compositionnels programmés ne doivent pas nécessairement aboutir à la réalisation immédiate d'une oeuvre achevée unique. Il est possible de leur donner uniquement une existence virtuelle, de les laisser ouverts à différentes réalisations. La possibilité apparaît alors, en plus de l'interprétation acoustique de l'oeuvre, d'une authentique interprétation compositionnelle de processus abstraits capables d'incarner des familles d'oeuvres diverses. La réalisation sonore étant faite immédiatement par un périphérique adéquat, contrôlé en temps réel et interactivement par l'ordinateur et le compositeur, ce dernier peut agir sur le fait à tous les niveaux, aussi bien à ceux de la structure compositionnelle générale qu'à ceux de sa réalisation détaillée. Plutôt qu'un objet arrêté, l'oeuvre est alors une virtualité abstraite dont une exécution incarne la synthèse avec une circonstance concrète - cf. les notions d'oeuvre ouverte et, surtout, d'oeuvre en mouvement, développées par Eco (1965). Le système PLAY décrit par Chadabe et Meyers (1978) constitue une ébauche de ce type de démarche, en ce qu'il comporte à la fois des processus compositionnels et de synthèse sonore en temps réel, tous contrôlables par l'interaction de trois catégories de directives : 1) des fonctions de contrôle précomposées, 2) des processus stochastiques, et 3 ) des dispositifs gestuels analogiquenumériques divers. On y trouve donc à la fois la programmation d'une structure abstraite précomposée, éventuellement la réalisation plus ou moins stochastique de cette structure, et enfin la possibilité cruciale de l'interprétation, aussi bien composition- 
nelle qu'acoustique : « ...le compositeur remplit deux différents rôles - d'abord celui de concepteur d'un système externe, puis celui de participant au fonctionnement de ce système " (Chadabe $1977: 11){ }^{18}$

Cette possibilité accentue davantage l'orientation conceptuelle de la composition musicale, que favorise de toute façon l'ordinateur :

Les systèmes impliquant un processus automatisé posent le problème de la conceptualisation de la composition en tant qu'activité séparée, parce que de tels systèmes engendrent de nouvelles données compositionnelles simultanément avec l'exécution du processus. De plus, chaque exécution sera spécifiquement différente. La musique produite par un tel système est identifiée davantage par la nature de son processus que par ses détails. Puisque les détails de musiques résultant d'un processus automatisé varieront d'une exécution à l'autre, l'attention délaisse les détails pour se fixer sur le processus lui-même, et sur le système produisant le processus. La conception du système est ainsi portée au premier plan de l'acte compositionnel. Cela rappelle le mot de Yeats : Comment peut-on distinguer le danseur de la danse? Le système et le processus musical qu'il produit sont reliés comme l'organisation est reliée au fonctionnement (ibid. : 7). ${ }^{19}$

La notion d'oeuvre musicale, ainsi que le rôle du compositeur dans son élaboration, sont totalement modifiés dans ce type de démarche :

La composition avec un système interactif en temps réel de processus automatisés constitue une expérience fort différente de la composition traditionnelle. ... En effet, il est peut-être opportun de se demander si le contrôle en temps réel de la composition,et la spécification détaillée de données, sont en pratique des procédés mutuellement exclusifs (ibid.). ${ }^{20}$

Quel que soit son degré d'influence musicale profonde, la synthèse sonore pose d'autre part de sérieux problèmes d'interfaces, c'est-à-dire de modes d'accès du compositeur aux divers niveaux des processus. On peut se contenter, en général, dans un contexte en temps différé, d'un accès alphanumérique par l'intermédiaire d'un terminal ordinaire - encore que des entrées graphiques ou gestuelles de certains types de données peuvent en 
assouplir l'utilisation. Cependant le temps réel rend cette question tout à fait critique, de par l'efficacité immédiate des interventions requises du compositeur. En plus des modes ordinaires de communication alphanumérique, différentes formes de graphisme doivent être adjointes (cf. Buxton 1978: 73 sq.; Buxton, et al. 1979; Laske 1978:44), et des dispositifs permettant de varier en temps réel des paramètres quelconques doivent être étudiés ergonomiquement. ${ }^{21}$

Outre ces questions de dispositifs matériels, l'élaboration des logiciels demande une grande attention : « ... nous avons observé que le design de la façade des systèmes est trop souvent négligé, et qu'ainsi seulement une faible part du plein apport d'un système se trouve réalisée " (Buxton 1978:1). ${ }^{22}$ S'il est nécessaire de maintenir un certain noyau d'information, bien adapté au fonctionnement interne de l'ordinateur et de ses périphériques, des programmes agissant en interface doivent en permettre l'accès musical suffisamment aisé. Afin de permettre l'intégration de divers types d'approches créatrices, ces programmes doivent être conçus idéalement dans un contexte de mise au point perpétuelle, permettant différentes représentations externes du noyau de données internes. Toute la souplesse nécessaire doit être préservée pour laisser le compositeur mettre en oeuvre une très large variété de processus compositionnels, eux-mêmes réalisés par des méthodes de travail personnelles et imprévisibles : " La composition interactive implique une variété de tâches et une variété de types de connaissance " (Laske $1978: 39$ ). ${ }^{23}$

\section{Approches de la synthèse sonore numérique}

La conséquence la plus fondamentale de la synthèse sonore numérique tient dans la liberté qu'elle procure. Le tout est de savoir que faire d'une technique aussi puissante, qui offre la possibilité de décrire en détail des ondes acoustiques quelconques. Mise à part la possibilité de reproduire tous les sons connus ou "naturels", si l'on veut produire des sons originaux, on a une latitude proportionnelle à la précision du processus : toute onde est possible, pourvu qu'elle n'exige pas de différencier plus de $2^{16}$ amplitudes discrètes (résolution), ni ne comporte de composantes de fréquences supérieures à $10000 \mathrm{~Hz}$. (moitié du taux d'échantillonnage), pour reprendre les valeurs mentionnées cidessus par exemple. Pour une seconde de son, l'on se trouve devant une grille immense de 20000 échantillons pouvant tous prendre l'une de $2^{16}$ valeurs ... Que pouvons-nous y faire? ${ }^{24}$ 
Si l'on examine la succession des échantillons en termes de simple théorie de l'information, deux extrêmes sont définis: d'une part l'information nulle, c'est-à-dire la répétition d'une valeur constante, et d'autre part l'entropie maximale d'une suite de valeurs imprévisibles, aléatoires. ${ }^{25}$ D'une part le silence, d'autre part le bruit blanc. Sans doute la musique est elle quelque part entre les deux? Il faut ajouter quelque chose au silence continu, ou retrancher quelque chose du bruit continu.

Pour notre perception, par contre, le bruit continu ne semble pas véhiculer plus d'information que le silence : ces deux extrêmes sont aussi blancs l'un que l'autre ${ }^{26}$ L'excès d'information est perçu, finalement, comme une continuité tout à fait redondante, d'entropie nulle. Notre attention fuit également ces deux extrêmes. L'oreille cherche des phénomènes auxquels se rattacher - périodicités, articulations, etc.-, des structures ordonnées, mais suffisamment imprévues tout de même, à la fois variées et redondantes. Il est nécessaire qu'une musique s'affirme en fonction d'un certain code partagé, soit connu d'avance par l'auditeur, soit établi par la pièce elle-même (cf. Kaegi 1971 : $24-26) .{ }^{27}$

Il faut donc produire des ondes acoustiques, des fonctions du temps, dans une plage adéquate de complexité. Dans l'optique que nous venons d'exposer, il y a pour ce faire deux méthodes en vérité, qui correspondent aux deux attitudes fondamentales que peut adopter un artiste dans sa tentative d'exploitation d'une réalité donée : "la suspension partielle du désordre ..., et la rupture partielle au sein d'un milieu très ordonné " (Riote 1974 : 13) ; soit sculpter des structures dans un phénomène brut et amorphe, soit élaborer des structures à partir d'éléments minimaux. Ces deux méthodes peuvent être pratiquées en outre selon deux approches : soit selon celle de théories acoustiques rendant compte des caractéristiques des événements sonores recherchés dans un cadre musical, soit selon celle d'une démarche de type compositionnel poussée au-delà d'événements sonores macroscopiques, jusqu'au niveau ultime des échantillons.

\section{a. Approches acoustiques}

Historiquement, la synthèse sonore numérique n'a nullement été conçue selon le point de vue absolu que nous venons d'esquiser. Max Mathews aborda cette méthode de génération sonore sous un angle très précisément acoustique (cf. Bowles 1970 : 15). Il a alors jeté les bases de tous les développements 
subséquents de la synthèse numérique, et plus particulièrement de ses approches acoustiques, qui sont à l'heure actuelle les mieux connues et les plus développées. La seule méthode praticable était à l'origine celle de l'élaboration des ondes sonores par des logiciels autonomes en temps différé. À partir du début des années 1970 , la technologie a permis la conception de périphériques spécialisés en temps réel, toutefois sans modification de l'approche fondamentalement acoustique incarnée par les premiers logiciels. Nous allons donc examiner brièvement cette approche acoustique de la synthèse sonore, en gardant présent à l'esprit qu'elle peut aujourd'hui être réalisée tout aussi bien par des logiciels que par des périphériques spécialisés.

L'archétype des logiciels de synthèse numérique est le système MUSIC V (Mathews 1969). ${ }^{28}$ C'est un ensemble de programmes acceptant des instructions préparées par le compositeur et spécifiant le mode d'élaboration de la structure acoustique des sons à produire. Ces instructions constituent une partition, dont la partie essentielle consiste en la définition d'algorithmes sonores (instruments, en terminologie MUSIC V courante).

Ces algorithmes sont construits à partir de modules élémentaires réalisant certaines opérations précises : il existe, par exemple, des modules oscillateurs (générateurs d'ondes acoustiques ou, plus généralement, de fonctions de temps décrites parl'utilisateur, à fréquences et amplitudes données), aléatoires (générateurs de fonctions de temps aléatoires obéissant à certaines normes données), additionneurs (mélangeurs de deux ou plusieurs composantes), multiplicateurs, filtres, etc. Par l'interaction de ces modules, on définit donc une suite d'opérations, ${ }^{29}$ d'injections de fonctions de temps suivies de divers traitements, aboutissant au signal sonore désiré. Les algorithmes ainsi constitués sont passifs : ce sont des circuits de modules incarnant des mécanismes acoustiques aptes à être excités en fonction de certains paramètres que l'utilisateur désirera varier selon un objectif musical.

Des algorithmes acoustiques de ce type peuvent réaliser les deux méthodes fondamentales de définition d'ondes acoustiques dont nous avons parlé ci-dessus : soit l'élaboration du son à partir de la combinaison de fonctions élémentaires simples, soit son extraction à partir de phénomènes amorphes. La première est parfois dénommée synthèse additive (cf. Moorer 1977: 5). Elle consiste donc à former le son désiré au moyen de l'action conjuguée de modules générant chacun des signaux simples (en dernier 
ressort : des sinusoïdes, par exemple), et à atteindre, par l'addition et l'interaction de ces composantes, le degré désiré de richesse et de complexité de timbre, de hauteur, d'intensité, de variations dynamiques de ces caractéristiques, etc. ${ }^{30}$ La seconde méthode est issue de recherches effectuées à l'origine sur la synthèse de la parole (cf. Moorer 1977 : 15 sq.; Cann 1979a, 1979b, 1980), et consiste, au moyen du filtrage de signaux riches constants, ${ }^{31}$ périodiques ou bruiteux, en l'extraction d'ondes acoustiques musicalement structurées et caractérisées.

Quoi qu'il en soit, cette approche de la synthèse remonte toujours à des modèles acoustiques précis des sonorités recherchées, à leur composition à partir d'ingrédients acoustiques, finalement à leur spectre de composantes élémentaires. Dans cette optique, la théorie de Fourier fournit un outil mathématique puissant, permettant de formuler précisément la composition de tout signal. ${ }^{32}$ En synthèse sonore numérique, la difficulté soulevée dans le cas de sons musicaux dotés de constantes variations dynamiques et de régimes transitoires complexes, par le fait que la théorie de Fourier ne rend compte, strictement, que d'ondes absolument périodiques et de durée infinie, ${ }^{33}$ peut être résolue en pratique par le recours au procédé si typiquement informatique de l'échantillonnage, encore une fois, qui consiste à considérer la continuité du son comme une juxtaposition suffisamment fine de spectres discrets. ${ }^{34}$ Avec de tels moyens, il est possible de procéder à la synthèse d'un événement sonore quelconque, pourvu que l'on sache décrire son mécanisme de formation et que l'on aie la possibilité de contrôler les paramètres de sa réalisation.

Pour compléter la partition destinée à la synthèse sonore, on ajoute à la définition des algorithmes un ensemble d'instructions destinées à activer ces processus passifs, en fonction de caractéristiques musicales. Ces instructions (énoncés NOTE en MUSIC V) remplissent la même fonction que la partition traditionnelle de musique instrumentale : elles décrivent la structure musicale de l'ensemble des sons à synthétiser, c'est-à-dire qu'elles spécifient les caractéristiques individuelles (par exemple : hauteur, intensité, timbre, etc.) de différents événements sonores - éventuellement engendrés par un même algorithme-, et qu'elles incarnent leurs inter-relations musicales - en termes de chronologie, de polyphonie, etc.

Les logiciels du type MUSIC $\mathrm{V}$, tout aussi bien que les logiciels de contrôle de périphériques en temps réel, sont donc 
conçus pour permettre au compositeur, avec le minimum de compromis et le maximum de liberté, de procéder à l'assemblage d'algorithmes à partir des modules disponibles, puis à leur activation sonore. Bien qu'elle puisse être contournée dans une certaine mesure, leur optique fondamentale consiste en la simulation numérique de processus ou de phénomènes acoustiques et/ou physiques. Ils partagent en outre une tendance essentielle, qui est de concevoir l'algorithme sonore comme un processus autonome, générateur d'événements discrets, caractérisés et individualisés, que le compositeur dispose, agence et transpose dans son oeuvre en fonction de critères compositionnels - souvent étrangers, en pratique, aux critères acoustiques gouvernañt la synthèse ellemême.

Mis à part le fait que le compositeur doit définir lui-même les algorithmes sonores qu'il utilise, ce type d'approche, par mise en situations musicales d'événements sonores basés sur des modèles acoustiques, s'inscrit tout à fait dans notre conception traditionnelle de la composition musicale. Une inter-relation profonde peut bien sûr s'établir entre la définition des algorithmes sonores et leur utilisation musicale, mais il y a toujours juxtaposition de processus de deux différents types : d'abord l'élaboration d'une structure proprement compositionnelle affiněe jusqu'au niveau des caractéristiques musicales d'événements sonores, dont la réalisation est ensuite déléguée à des mécanismes d'une autre nature.

Joel Chadabe $(1977: 8)$ voit dans cette situation une hiérarchie de trois niveaux fonctionnels temporels - de fonctions de temps, pourrait-on dire :

1) le temps acoustique (audio-time), qui est celui du fonctionnement acoustique des algorithmes sonores, dans des durées de l'ordre de la milliseconde, c'est-à-dire du calcul des échantillons ;

2) le temps conscient (conscious-time), qui correspond au niveau des énoncés NOTE activant les algorithmes, imposant au niveau précédent des structures de l'ordre de la seconde ;

3) le macro-temps (longterm-time), qui vient coiffer les deux précédents, correspondant lui-même sans doute à une certaine hiérarchie de niveaux emboîtés d'organisations compositionnelles, structurant le temps conscient. ${ }^{35}$

Il faut noter que dans cette hiérarchie, cependant, l'énoncé NOTE dont il a été question ci-dessus occupe une position ambiguë. L'algorithme qu'il active peut posséder le pouvoir d'en- 
gendrer un événement sonore beaucoup plus complexe qu'une note - au sens instrumental traditionnel, ou ce que Moles (1972: 172) dénomme un objet sonore $:^{36}$ la synthèse sonore permet de déclencher des événements pouvant aller jusqu'à affirmer leur propre structure au niveau du macro-temps, englobant éventuellement plusieurs événements subordonnés perceptivement discernables. Comme on l'a vu ci-dessus, la seule caractéristique essentielle de l'énoncé NOTE est une certaine autonomie fonctionnelle : l'activation d'un algorithme sonore en fonction d'un contexte musical. ${ }^{37}$

Dans une analyse hiérarchique similaire de la perception sonore, Abraham Moles (ibid. : 182) distingue un quatrième niveau, que l'on peut concevoir comme plus fin que le temps acoustique, ou inclus dans celui-ci : le niveau des structures élémentaires, dont il dit qu'elles se situent "dans l'épaisseur du présent "- pour les trois niveaux décrits ci-dessus, Moles emploie la terminologie suivante : micro-structure, structure intermédiaire et macro-structure (ibid. : 182-83). À l'occasion du développement du concept de quanta de sensation sonore (ibid. : 28 sq.), Moles définit l'épaisseur du présent comme un «seuil de perception ... en deçà [duquel] tous les phénomènes se confondent dans une simultanéité psychologique " (ibid. : 31). Il en arrive ainsi à préciser la notion de " grain de son, ${ }^{38}$ l'atome sonore le plus petit imaginable ... représenté par [un volume tridimensionnel] ayant pour côtés le seuil différentiel de fréquence $\Delta F / F$, le seuil différentiel d'intensité $\Delta \mathrm{L} / \mathrm{L}$, le seuil différentiel de durée $\Delta \mathrm{T} / \mathrm{T}$ » (ibid. : 32).

Par des expériences psychoacoustiques sur ces trois seuils de différentiation sonore, on peut ainsi délimiter des signaux élémentaires qui représentent, dans le cadre d'une analyse informationnelle de la perception, des quanta d'information, c'est-àdire des événements sonores élémentaires, minimaux. ${ }^{39}$ L'idée d'exploiter musicalement de tels " grains élémentaires ", micronotes ou micro-sons, ${ }^{40}$ a été énoncée et mise en oeuvre par Xenakis dans le chapitre II de Musiques formelles :

Tout son est une intégration de grains, de particules élémentaires sonores, de quanta sonores. Chacun de ces grains élémentaires a une triple nature : la durée, la fréquence et l'intensité. Tout son, toute variation sonore même continue est conçue comme un assemblage de grains élémentaires suffisamment nombreux et disposés dans le temps d'une façon adéquate. Donc : tout complexe sonore est analysable 
en séries de sons purs sinusoïdaux même si les variations de ces sons sinusoïdaux sont infiniment rapprochées, brèves et complexes. Dans l'attaque d'un son complexe, dans son corps, dans sa chute, des milliers de sons purs apparaissent dans un intervalle de temps $\Delta t$ assez court (1963:61).

Il s'agit d'une extension musicale des considérations des théories de Fourier et de l'information, mais qui est dans l'esprit de l'application de principes quantiques pour l'analyse de phénomènes biochimiques ou physiologiques liminaux ou subliminaux (cf. Goudot 1952) - en l'occurrence il s'agit de la perception sonore, étudiée par Gabor (1947). Comme l'indique la citation d'un modèle acoustique, et de l'hypothèse de pouvoir les mettre en oeuvre pour synthétiser des sons quelconques. ${ }^{41}$ L'approche de la synthèse sonore par événements sonores discrets, par notes autonomes, est ainsi poussée à son degré de finesse ultime, et les structures hiérarchiquement supérieures, correspondant à des organisations acoustiques perceptibles, c'est-à-dire composées d'ensembles de micro-sons, doivent être alors prises en charge par des structures compositionnelles adéquates, agissant au niveau de l'activation d'événements sonores structurés, de l'ordre du temps conscient.

\section{b. Approches non-acoustiques}

Les approches que nous allons décrire maintenant peuvent être rassemblées globalement sous l'expression composition directe. Ces termes signifient en effet, s'il n'est pas question de faire fi de la fonction acoustique des échantillons d'ondes acoustiques, qu'il est toutefois concevable de pousser une démarche compositionnelle ou un processus algorithmique quelconquejusqu'au niveau ultime du calcul des échantillons, sans recourir nécessairement à des modèles acoustiques prenant, à partir d'un certain niveau, le relais de modèles musicaux ; bref, qu'il est possible de composer directement le son même, c'est-à-dire le train d'échantillons acheminés vers la conversion numériqueanalogique.

Nous avons vu que le recours à des quanta sonores élémentaires se situe à la frontière de la composition traditionnelle et de la composition directe, car il s'agit en effet d'une micro-composition basée sur un modèle acoustique minimal. D'autre approches se situent près de cette frontière, qui permettent au compositeur de dessiner le détail des ondes de pression. ${ }^{42}$ Mais la composition directe est une démarche beaucoup plus radicale, principalement 
en ce qu'elle englobe la possibilité de ne pas recourir à l'exploitation périodique d'ondes prédéterminées et concourant à la synthèse d'événements sonores autonomes que la composition se limite à transposer pour leur permettre de remplir diverses fonctions musicales. À la limite, la composition directe dissout entièrement la notion même d'événement sonore. Les caractéristiques définissant cette notion - hauteurs et profils mélodiques, structures dynamiques, etc., qui sont essentiellement liées à l'introduction de phénomènes périodiques et à l'imposition de fonctions de contrôle macroscopiques - peuvent être en effet outrepassées par un travail compositionnel appliqué au niveau des échantillons sonores eux-mêmes. Sur un petit groupe d'échantillons, de telles notions n'ont plus leur place ; elles sont en réalité impossibles. Si des événements sonores perceptuellement distincts et caractérisés se réalisent dans un tel contexte, ce ne peut être dû qu'à l'effet de structures supérieures agissant sur un grand nombre d'échantillons ; et c'est justement à ce niveau que la composition directe cherche à se libérer des modèles acoustiques.

Une fois acquise la volonté d'étendre la démarche compositionnelle au-delà des organisations macroscopiques, l'optique est tout à fait autre. On fait radicalement table rase, et l'unique problème à résoudre, qui touche en réalité au plus profond de la nature acous tique de la musique, s'énonce simplement : il s'agit de fabriquer des variations de pression atmosphérique dans le temps. D'un point de vue absolu, la musique n'est pas autre chose, et l'informatique permet de la saisir à bras-le-corps sous cet aspect entièrement nu. La composition musicale se réduit à un travail direct sur les deux dimensions de cette grille d'échantillons dont nous parlions ci-dessus : le temps et l'amplitude.

Le caractère radical de cette approche ne modifie pas pour autant la nature essentielle du travail compositionnel ; elle en représente une extension. La nécessité de l'interaction hiérarchique de plusieurs niveaux d'organisations demeure, avec toujours la prépondérance d'une certaine échelle chronologique intermédiaire - le temps-conscient (cf. ci-dessus) :

Les changements qui définissent et éclairent le mieux une composition musicale ne se trouvent pas au niveau le plus bas, ni au plus élevé de la hiérarchie, mais quelque part entre les deux. Tandis que se déroule la musique, nous ne percevons consciemment ni les impulsions ${ }^{43}$ individuelles, qui évoluent trop rapidement, ni la forme globale de l'oeuvre, qui 
évolue trop lentement. La musique est véhiculée dans le temps conscient, à l'intérieur duquel nous avons conscience de notre conscience du changement (Chadabe $1977: 9$ ). ${ }^{44}$

Cette nécessité se trouve même soulignée par la composition directe, car la macro- et la micro-composition peuvent y être intégrées en une démarche unique.

Nous avons vu que deux méthodes essentielles sont envisageables pour remplir cette grille d'échantillons sonores dans le temps, par des ondes de pression situées dans une certaine plage de complexité intermédiaire : d'une part des processus vers l'amont - prenant comme point de départ des phénomènes sonores excessivement complexes afin de leur imprimer des structures perceptibles, c'est-à-dire d'en retrancher un excès d'information; d'autre part un travail vers l'aval - élaborant ex nihilo les structures acoustiques et musicales recherchées. Il est vraisemblable que l'objectif musical à atteindre implique un édifice complexe de plusieurs niveaux hiérarchiques interdépendants et emboîtés, dont l'élaboration donnerait éventuellement lieu à l'utilisation conjointe de ces deux méthodes opposées.

Ce genre de synthèse sonore étant par définition entièrement libre, on retrouve naturellement une forte empreinte personnelle dans les expériences auxquelles se sont livrés quelques expérimentateurs. Les compositeurs y voient donc la possibilité de l'extension de leurs propres processus compositionnels, et de l'intégration totale de la musique, tandis que des méthodes algorithmiques révèlent chez d'autres une conception sonore radicalement informatique, tout à fait nouvelle dans notre contexte musical.

Xenakis (1971 : 246-49) énumère quelques méthodes de synthèse basées essentiellement sur la distribution stochastique des amplitudes des échantillons, soit de façon directe et pure, soit par la combinaison de diverses variables aléatoires, ou par la mise en oeuvre d'une hiérarchie de fonctions aléatoires ou de contrôles de type compositionnel : s'il est possible d'utiliser purement une distribuiton quelconque pour obtenir une sonorité, ${ }^{45}$ la nécessité se fait rapidement jour de varier les sonorités, puis de leur imposer une articulation musicale. Xenakis a réalisé d'autre part quelques expériences de direction opposée, basées sur l'exploitation d'une période dont la forme évolue graduellement par l'application de distorsions aléatoires cumulatives sur une période de départ - éventuellement très simple : sinusoïdale par exemple (Xenakis 1978). 
Pour sa part, Koenig a mis au pont un système de synthèse incarnant au niveau des échantillons sonores des processus compositionnels identiques ou apparentés à ceux mis en oeuvre dans les algorithmes de composition instrumentale dont il est l'auteur. ${ }^{46}$ Le S.S.P. - Sound Synthesis Program (Berg, et al. 1980) en effet, permet de définir des ondes de pression par une combinaison d'opérations de synthèse aléatoire et de manipulation de données préexistantes, effectuées sur des échantillons isolés ou sur des segments de périodes. ${ }^{47}$

Le même type de démarche est mis en oeuvre par Herbert Brün dans son Project Sawdust (Blum 1979 : 6) : des éléments d'échelles chronologiques diverses, depuis le niveau des échantillons sonores jusqu'à celui d'événements déjà constitués acoustiquement, sont manipulés directement, et la structure compositionnelle s'élabore donc hiérachiquement à partir d'éléments réduits donnant naissance à des éléments plus longs, etc. Brün est sans doute l'un des premiers à avoir conçu l'idée d'un processus de composition directe, pour sa pièce Infraudibles, dès avant 1967; il s'est exprimé clairement là-dessus :

En remplaçant la modulation de [hauteurs] par une [combinatoire] de périodes, le contrôle de la chronologie et des durées, que le compositeur applique aux macro-événements de sa composition, peut être aussi étendu à l'infrastructure des sons formateurs d'événements. Ainsi la hauteur devient un résultat de la composition, au lieu de fonctionner comme [l'un de ses] éléments. Le même raisonnement s'applique au concept de timbre sonore (cité in Hiller $1970: 63$ ). ${ }^{48}$

Si les quelques expériences de composition directe que nous venons de décrire sont, au fond, essentiellement traditionnelles - en ce qu'elles représentent des extensions de techniques compositionnelles éprouvées-, il est aussi possible d'at taquer directement la micro-structure sonore par l'application d'algorithmes quelconques déterminant les valeurs successives des échantillons. Paul Berg (1979) poursuit des expériences dans cette voie radicalement informatique, en acheminant vers la conversion numérique-analogique les contenus successifs d'un registre de l'unité arithmétique et logique d'un ordinateur pendant l'exécution d'un algorithme. ${ }^{49}$ Dans un tel processus de synthèse instructionnelle (instruction synthesis), iln'y a pas forcément de relation simple et directe entre la nature ou la fonction apparente de l'algorithme exécuté et la forme de l'onde sonore résultante. ${ }^{50}$ 
Parce que de cette manière elle dissocie en quelque sorte les données du résultat, cette méthode est, dans un premier temps, essentiellement pragmatique : le son est considéré comme un simple phénomène bidimensionnel que l'on doit produire au moyen d'un ordinateur, peu importe comment. ${ }^{51}$ Mais il y a, derrière la brutalité de cette approche, l'idée d'ouvrir de nouveaux domaines de sonorités et de structures musicales :

Explorer les capacités idiomatiques de l'ordinateur dans le domaine de la synthèse sonore ... des systèmes où l'ordinateur est essentiel pour une raison autre que celle de l'ampleur de la tâche. Où [l'ordinateur] pourrait contribuer à la production de nouvelles sortes de sons. Ou de processus de production sonore. Ou au moins de nouvelles représentations du son (Berg 1975, cité par Holtzman $1979: 54$ ). ${ }^{52}$

Et Berg écrit ailleurs, plus poétiquement : «Entendre ce qui, sans l'ordinateur, ne saurait être entendu ; penser ce qui, sans l'ordinateur, ne serait pas pensé ; apprendre ce qui, sans l'ordinateur, ne serait pas appris » $(1979: 30) .{ }^{53}$

La synthèse sonore numérique est donc une technique qui offre une très large liberté, et dont les implications s'étendent depuis le niveau strictement physique du son, jusqu'aux plus hautes ramifications compositionnelles et proprement musicales. À l'intérieur des contraintes du processus de conversion numériqueanalogique, toute onde de pression est possible - et, espérons-le par extension, toute musique ${ }^{54}$ - pourvu cependant que l'on sache comment la décrire ou l'obtenir. Cette immense liberté est un stimulant aigu. Elle nous révèle souvent, d'une façon quasi métaphysique, que nous ne savons justement pas qu'en faire; elle met à jour les insuffisances de nos modèles - acoustiques ou autres - pour la création musicale, et nous dévoile brutalement l'énorme complexité, souvent insoupçonnée, des phénomènes sonores, et de la musique à plus forte raison; car bien sûr toute liberté est une responsabilité, et, dans ce domaine, rien n'est donné d'avance : de l'ordinateur, l'on n'obtient malheureusement que ce que l'on a spécifié, et rien d'autre ... : 
La synthèse sonore par ordinateur est un medium vous permettant d'avoir ce que vous spécifiez; vous obtenez tout cela et rien que cela. Au moins, l'on est toujours dans la position de savoir ce que l'on a, que cela soit ce que l'on voulait ou non (Howe $1975: 170$ ). ${ }^{55}$

\section{NOTES}

1. Pour plus de détails techniques, le lecteur est renvoyé aux excellents articles suivants: Moore (1978a et 1978b), Moorer (1977 et 1978).

2. Cet article est extrait d'une thèse de doctorat présentée par l'auteur à l'Université de Paris I, sous la direction de M. Iannis Xenakis.

3. Il est plaisant de souligner que dans la perception du son, l'oreille interne aura retransformé les variations continues captées par le tympan en d'autres impulsions discrètes (décharges neuroniques) acheminées vers le cerveau. De toute façon, ces distinctions discret-continu sont avant tout une affaire d'échelle d'observation : ce qui nous semble perceptivement continu peut très bien être discret au niveau moléculaire, atomique ou sub-atomique.

4. En termes de stricte transmission d'information, un taux de $f$ échantillons/seconde permet de rendre compte de toute fréquence inférieure ou égale à $f / 2$ (cf. Steiglitz 1974 : 44). Cependant, l'échantillonnage ne donne alors qu'une simple image binaire des ondes de fréquences fondamentales voisines de $f / 2$. Pour des besoins musicaux, la limite supérieure des fréquences utilisables est environ 0.7 $f / 2$, ce qui donnerait $7000 \mathrm{~Hz}$. dans le cas de 20000 échantillons par seconde. Pour la synthèse, cette limite représente à peu près un minimum acceptable. Pour la reproduction fidèle de sons réels enregistrés, cependant, il est nécessaire d'augmenter jusque vers 40000 échantillons/seconde et au-delà.

5. Par exemple : une résolution de 16 bits - c'est-à-dire permettant la représentation de $2^{16}(65536$ ) valeurs différentes d'un échantillon. Une résolution insuffisante, par contre, peut être assimilée à une distorsion excessive induisant un bruit; on peut compter sur un peu plus de 6 décibels de rapport signal/bruit par bit de résolution : $201 \log _{10}{ }^{2}=6 .{ }^{02}$ (Mathews 1969 : 7). Un calcul plus précis de cette distorsion doit tenir compte de l'onde acoustique synthétisée (ibid : 25).

6. Exception faite des procédés de synthèse inst ructionnelle décrits en II.2 ci-dessous (Berg 1979 ; Holtzman 1979), qui sont justement basés sur l'idée d'exploiter la configuration matérielle concrète d'un ordinateur. Dans des limites assez étroites, certains autres processus de synthèse sont cependant à la portée temps réel des ordinateurs modernes - cf. le système POD par exemple (Truax 1977).

7. En ce sens, on pourrait soutenir qu'il n'y a pas de différents degrés de temps différé. Il y a seulement une frontière nette entre le temps réel et le temps 
différé, pour tout ce qui concerne l'attitude musicale et compositionnelle de l'utilisateur. Idéalement, une fois qu'il a accepté le principe d'une attente, l'utilisateur peut, en effet, faire preuve d'une grande patience.

8. Cette écriture peut être éventuellement un dessin (cf.le système UPICdu CEMAMu, note 42 ci-dessous), mais ce n'est pas là une différence fondamentale.

9. Il existe un très grand nombre de tels appareils, expérimentaux ou commercialisés. Il serait fastidieux de dresser ici une liste de références précises ; qu'il suffise de recommander la lecture du Comptuer Music Journal. On ne trouvera presque pas, dans cette revue, de descriptions de systèmes hybrides ordinateurs contrôlant des périphériques analogiques, dont l'exemple le plus classique est le système GROOVE (Mathews \& Moore 1970), et qui constituent une réponse technologique différente, historiquement antérieure mais encore viable dans certains cas, aux inconvénients du temps différé.

10. Ce type d'appareil plus récent est moins répandu que le précédent. Voir Allouis (1976; 1979), Moorer, et al. (1979) et Wallraff (1979). Quant à la machine 40 dont il est question dans l'article de Moorer, et. al., elle est maintenant supplantée par la $4 \mathrm{X}$ dont elle était un prototype de mise au point. Technologiquement, le domaine de la synthèse sonore bouge rapidement, et le lecteur comprendra aisément qu'entre la rédaction de cet article et sa publication, quantité de nouveautés auront vu le jour.

11. À titre indicatif, voici l'opinion d'une personne qui n'est pas directement impliquée dans le domaine musical, mais qui effectue des recherches dans celui des applications de l'informatique aux arts visuels - le peintre Harold Cohen:

"Je pense que la plupart des travaux [dans le domaine de l'informatique musicale] s'occupent primordialement de technologie. J'ai parfois l'impression qu'une formidable quantité d'énergie est consacrée à la création de l'arme musicale suprême - qui puisse produire tous les bruits qui ont jamais été produits et un bon paquet qui ne l'ont jamais été. " [ "I think of most work in [the domain of computer music] being involved with engineering primarily. I sometimes get the feeling there's an awful lot of energy going intocreating the ultimate musical weapon - that can make all the noises that have ever been made and quite a lot that haven't been " (Roads 1979b : 53) .]

12. "The real problems in computer music are not in the area of sound synthesis ... [Not] enough attention is being placed on the development of tools to aid the composer in controlling ... sounds in a musical context " .

13. Bien entendu, des processus compositionnels programmés interviennent forcément dans la composition directe des ondes acoustiques, ou peuvent être utilisés pour toutes formes de compositions, qu'elles soient destinées à l'exécution instrumentale ou à la synthèse sonore. Nous ne voulons pas traiter ici cette question sous l'angle purement compositionnel, mais plutôt examiner quelles seraient les influences de la synthèse sonore numérique proprement dite sur la composition musicale.

14. On peut classer dans une catégorie à part des logiciels permettant uniquement l'exécution en temps réel d'une partition précomposée. Quel qu'ait été le type de processus, algorithmique ou non, mis en oeuvre pour la préparation de la composition, il s'agit là d'une stricte interprétation. Buxton (1978:35-36) parle dans ce cas d'un mode d'utilisation de l'ordinateur situé entre deux extrêmes : le mode instrumental (Piano Mode) d'une part, et le mode enregistreur (TapeRecorder Mode) d'autre part. L'ordinateur off re la possibilitécruciale dela mémorisation des paramètres d'une interprétation, et de leur correction en temps réel: 
dans le système décrit par Buxton, et al. (1980:12-13), les paramètres accessibles sont le registre, le tempo, l'articulation, l'amplitude et la richesse du timbre. Le système GROOVE (Mathews \& Moore 1970) offre aussi à l'utilisateur la possibilité de programmer des actions sur de tels paramètres. Le compositeur a ainsi le loisir de parfaire une synthèse sonore en grand détail, avant de procéder à son enregistrement définitif, ou même éventuellement de l'interpréter en concert (cf. Risset $1971: 9-11$ ).

15. Sans entrer dans le détail de leurs différentes optiques et technologies, voici quelques exemples de tels systèmes : SSSP (Buxton 1978), GROOVE (Mathews \& Moore 1970), POD (Truax 1977). Il existe certainement toujours une interaction entre la composition et son résultat sonore, mais il faut avouer que les systèmes en temps différé le rendent parfois très difficile à établir en pratique.

16. On songe forcément aux systèmes de Computer Aided Design, aujourd'hui courants dans tous les domaines du design industriel, du génie mécanique, electronique, etc. (cf. Buxton $1978: 21$ ).

17. Cette situation est identique, bien entendu, dans le cas de la programmation d'algorithmes compositionnels autonomes, même sans recours à la synthèse sonore. Les innovations techniques provoquent souvent de tels déplacements du centre de gravité des oeuvres humaines ; pensons par exemple aux bouleversements provoqués par l'imprimerie, ou la photographie.

18. "... the composer functions in two ways - first as the designer of an external system, then as a participant in that system's functioning ". C'est-à-dire que le compositeur crée un système objet, puis est impliqué comme sujet participant au fonctionnement de cet objet.

19. "Process automation systems pose a problem in conceptualizing composition as a separate activity because such systems are generating new compositional data simultaneously with its performance. Further, every performance will be different in its specifics. The music produced by these systems is identified more by the nature of its process than by its details. Since the details of process automation music will vary from performance to performance, one's attention shifts from the details for the process itself and to the system which produces the process. Designing the system thus comes into focus as the first act of composition. It brings Yeats' words to mind: How can we know the dancerfrom the dance? The system and the musical process it produces are related as organization is related to functioning " .

20. "Composing with a realtime interactive process automation system is an experience significantly different from traditional composing. ... Indeed, it might be appropriate to ask if realtime control over composition and detailed specification of data are in fact mutually exclusive procedures » .

21. Input Devices : claviers, potentiomètres, boutons, manettes bi- ou tridimensionnelles, écrans, surfaces sensibles au toucher, etc. (cf. Chadabe 1977: 8). Le terme ergonomique est pris dans son sens restreint : adaptation de la machine à l'homme, recherche d'une communication ef ficace entre l'utilisat eur et l'outil ; il va sans dire que n'entrent pas ici de considérations de rendement au sens industriel.

22. "... it is our observation that the front end engineering of systems is too often neglected, thereby causing only a small portion of the system's full benefit to be realized".

23. "Interactive composition involves a variety of tasks and a variety of kinds of knowledge». 
24. Pierre Barbaud a utilisé cette image heureuse : «Terra ignota ubi sunt leones".

25. Engendrées selon une distribution uniforme discrète dans l'intervalle $\left[0,2^{16}\right]$. On a alors, bien entendu, 16 bits d'entropie (Cullmann, et al.1970:96); ceci toujours avec un convertisseur numérique-analogique 16 bits à titre d'exemple.

26. Dans l'esprit de la théorie de l'information, voici quelle serait l'antithèse de la fameuse pièce absolue de Cage, 4' $33^{\prime \prime}$ pour piano silencieux - dont l'essentiel tient dans la mise en scène du silence et son écoute attentive, pendant laquelle se produiront "des sons non voulus par le compositeur " (Charles 1978: 261) : 4'33" d'un bruit blanc continu à puissance maximale, afin que personne n'entende autre chose! Musicalement, cependent, l'opposition n'a pas tout son sens : le bruit constant n'apporte aucune information, n'éveille aucun intérêt en soi, pas plus que le silence.

27. La musique n'est pas intégralement réductible à ce schéma informationnel. En l'occurrence, ce point de vue théorique ne permet que de « préciser les limites inférieures (banalité) et supérieures (complexité) du débit d'information nécessaires à la perception d'une forme intelligible " (Pasquet $1979: 3$ ).

28. Tel que décrit par Mathews (1969), MUSIC V est le fruit d'une élaboration progressive étendue sur une dizaine d'années. MUSIC V a continué à évoluer par la suite (cf. Richer 1979) et partage d'autre part des racines communes avec d'autres systèmes analogues : MUSIC 360 (Vercoe 1971), MUSIC4BF (Howe 1975), MUSIC10 (Battier 1980), MUSIC11 (aujourd'hui commercialisé).

29. Le mode d'assemblage des modules ne constituait pas, à l'origine de MUSIC V, à proprement parler un langage, au sens informatique, c'est-à-dire une syntaxe permettant la rédaction de programmes - dont l'essence tient dans l'inclusion d'énoncés conditionnels (SI ... ALORS ... SINON ..., etc.) permettant l'autonomie décisionnelle des algorithmes, Toutefois, le système MUSIC10 inclut la possibilité d'une authentique programmation de type ALGOL. D'autre part, l'inclusion récente, dans MUSIC $V$, de modules décisionnels et d'un décodeur syntaxique élaboré, permet aussi, jusqu'à un certain point, la véritable programmation (cf. Richer 1979; Haynes 1980).

30. La méthode additive la plus stricte consiste à synthétiser un son à partir des composantes sinusoïdales de son spect re obtenu par transformée de Fouriercela suppose bien sûr que l'on connaît déjà la description phy sique du son à obtenir ... Il y a là un cercle vicieux que le compositeur ne peut rompre qu'en risquant quelque chose. Mais on peut grouper dans cette catégorie d'autres processus apparentés, qui sont primoridalement des raccourcis algorithmiques. Par exemple : la modulation de fréquence (Chowning 1973) et la distorsion non-linéaire (waveshaping) (Arfib 1978 ; Roads 1979a). Des processus de ce type permettent l'accession à des domaines de spectres à l'intérieur desquels il est possible d'effectuer des variations dynamiques de timbre de façon relativement aisée. On sait cependant que l'intérêt d'un son - du moins son intérêt musical - tient dans de subtiles variations de spectre et quantité de phénomènes transitoires. Pour rehausser l'intérêt des sons composés d'un spectre trop constant, pour leur insuffler une apparence non-synthétique, il faut introduire dans la synthèse des masses de contrôles annexes - considérables par rapport à la subtilité des effets à obtenir. Il faut ainsi accéder à un niveau de complexité tel que les données nécessaires sont pour le moment difficiles à cerner et à manipuler.

31. C'est-à-dire une matière première musicalement neutre, ou amorphe, comme on l'a vu, imitant en cela les mécanismes physiologiques de la phonation. 
Mais ce type de processus soustractif peut aussi bien être réalisé sur une matière déjà structurée (cf. Cann 1979a : 6 et Moorer $1977: 16$ ) ; on a alors imposition des structures de filtrage sur celles de la matière originale. Ajoutons que ce type de synthèse est réalisé par des systèmes spéciaux, et qu'il serait difficilement accessible à des systèmes du type MUSIC $V$, orientés primordialement vers les méthodes additives.

32. Il existe d'autres méthodes mathématiques utilisables. Par exemple : les fonctions de Walsh (Rosenberg 1979), qui présentent l'avantage d'être des fonctions rectangulaires, mieux adaptées à l'informatique que les fonctions trigonométriques de Fourier. Cependant la théorie de Fourier possède la supériorité de correspondre plus exactement à nos schèmes perceptifs et d'appréhension intuitive ou musicale des phénomènes acoustiques complexes. À la limite, quel que soit le modèle utilisé pour la synthèse d'un son, il est toujours utile de revenir à la théorie de Fourier pour comprendre ce que l'on fait (Risset, communication personnelle, 1978 ; cf. aussi l'exemple de Berg, et al. 1980 : 31 sq.).

33. "Mais l'analyse de Fourier est une description intemporelle, en termes d'ondes exactement périodiques de durée infinie. " [ "But Fourier analysis is a timeless description, in terms of exactly periodic waves of infinite duration " (Gabor 1947 : 591).]

34. On a même le loisir d'interpoler, si nécessaire, entre ces spectres discrets. Ce procédé représente bien sûr une entorse à la théorie, et suppose une inexactitude proportionnelle à la petitesse de la fenêtre d'analyse spectrale extraite du signal continu (cf. Moore 1978b : 49). L'inconvénient pratique de cet échantillonnage spectral est qu'il implique un accroissement considérable de la masse des données requises pour la synthèse (cf. Moorer 1977 : 12).

35. Dans une optique légèrement différente, c'est-à-dire orientée primordialement vers l'analyse des structures mentales mises en jeu par l'activitécompositionnelle dans un contexte de synthèse sonore - tandis que Chadabe part d'un point de vue pragmatique - Otto Laske (1978: 40-41) reprend cette même hiérarchie, en désignant toutefois son niveau supérieur par interpretive-time plutôt que longterm-time.

36. Moles $(1972: 172)$ donne trois caractéristiques de l'objet sonore : homogénéité, individualité, évolution. Cet objet est proche de la note instrumentale traditionnelle. Pierre Schaeffer (1977 : 268 sq. en particulier) utilise l'expression objet sonore dans une toute autre acception, plus générale ; c'est pourquoi nous préférons utiliser ici la désignation événement sonore, plus neutre, mais qui correspond, au moins au niveau de l'échelle de sa fonction musicale, éventuellement tout à fait variable, à l'objet sonore schaefferien.

37. «La notion de note musicale [a été] pulvérisée " par l'électroacoustique et la synthèse sonore (Revault d'Allones $1973: 37$ ), bien qu'elle semble survivre dans le vocabulaire courant de la synthèse sonore numérique. L'énoncé NOTE représente pourtant bien "l'unité syñtaxique du discours musical " (Nattiez 1975 : 196), une unité " possédant un début et une fin » (ibid.: 115); mais il peut correspondre à un amalgame acoustique encore plus complexe que la note traditionnelle (ibid.: 254), à tout un ensemble d'événements perceptibles. Nous sommes loin du temps où, selon Laloy (1909:49), "le sentiment de la musique [était] dans les notes elles-mêmes, non dans les nuances du timbre qui [étaient], bien qu'on [sentît] le charme, strictement accessoires "; dans une telle phrase, la signification du mot note se réduit pratiquement au seul symbole graphique disposé sur la portée : une hauteur fixe et précise, dotée d'ue certaine 
durée. Notons aussi que, tout autant qu'à celui de la macro-structure, l'énoncé NOTE peut agir au niveau de la micro-structure. Si sa fonction algorithmique est toujours claire, sa fonction musicale est ambiguë puisqu'elle peut naviguer librement parmi tous les niveaux de Chadabe.

38. C'est nous qui soulignons.

39. Cette idée remonte à Gabor, qui fait une analyse bidimensionnelle (temps $\mathbf{x}$ fréquence) des phénomènes sonores pour définir des " signaux élémentaires [qui sont] des oscillations harmoniques ... modulées par une impulsion [en forme de courbe de Gauss]. " [ " elementary signals [which] are harmonic oscillations ... modulated by a probability pulse " (1947: 592) — cf. aussi Pimonow (1962: 74-76).] Dans l'espace temps $\mathrm{x}$ fréquence, de tels signaux élémentaires occupent, en fonction de l'inégalité de Schwarz, une aire minimale

\section{$\Delta t \Delta f \leqslant 1$}

(ibid., 591). Cette analyse correspond, dans la théorie de Fourier, à l'impossibilité de dissocier les deux dimensions considérées : le temps et la fréquence. Bracewell (1965:160) énonce le fait ainsi:

"On peut spécifier des fonctions de temps arbitraires ou des spectres arbitraires, mais non les deux à la fois. Ainsi une aire finie du plan temps-fréquence ne peut contenir qu'un nombre fini de données discrètes indépendantes. Le produit largeurde-bande-passante - durée d'un signal ne peut être inférieur à sa valeur pour un signal élémentaire ne contenant qu'une donnée discrète. Un tel signal peut être bref et de bande passante étendue, ou quasi monochromatique et persistant. Puisque les largeurs équivalentes d'une fonction et de sa transformée sont des valeurs réciproques, il s'en suit que

durée équivalente $x$ largeur de bande passante équivalente $=1$. "

[ "One may specify arbitrary functions of time or arbitrary spectra, but not both together. Thus a finite area of the time-frequency plane can contain only a finite number of independent data. The bandwidth-duration product of a signal cannot be less than the value for an elementary signal containing only one datum. Such a signal may be brief and wide-band or quasi-monochromatic and persistent. Since the equivalent widths of a function and its transform are reciprocals, it follows that

$$
\text { equivalent duration } \mathrm{x} \text { equivalent bandwidth }=1 \text {." ] }
$$

Gabor justifie ensuite par la psychoacoustique l'utilité de ce modèle : «Mach a montré que si une oscillation sinusoïdale n'est émise que pendant quelques cycles seulement, l'oreille l'entendra comme un bruit, tandis qu'après une durée minimum ... elle sera entendue comme une courte note musicale, de fréquence définie. " [ "Mach showed that if a sinusoidal oscillation is sounded for a few cycles only, the ear will hear it as a noise, while after a minimum duration ... it will be heard as a short musical note, with ascertainable frequency" (1947: 592). ] En résumé, le son minimal défini ci-dessus représente bien une particule sonore irréductible, dont la perception peut varier cependant : si l'on ne se limite pas à la seule épaisseur du présent de Moles, l'oreille percevra la particule avec une largeur de bande inversement proportionnelle à sa durée. $\mathrm{Au}$ moyen donc de la seule variation de leur durée, on peut utiliser des "fonctions sinusoïdales ayant comme enveloppe une courbe de Gauss" (Xenakis $1963: 61$ ) pour produire des effets situés entre deux extrêmes : d'une part des sons purs prolongés, d'autre part des impulsions bruiteuses. Dans l'analyse de Moles et de Xenakis, une troisième dimension - l'intensité - est adjointe au temps et à la fréquence. Avec l'ajout 
d'autres dimensions - le timbre et la localisation spatiale - un type de son issu de cette particule sonore élémentaire est exploité systématiquement dans notre pièce pour haut-parleurs Les portes du sombre Dis, réalisée à l'IRCAM en 1979.

40. Moles (1972: 182) utilise le terme microson dans une autre acception, signifiant pratiquement " détail d'un son capté par un microphone».

41. Il semble cependant que l'inter-relation fondamentale temps $\leftrightarrow$ fréquence soulignée par Gabor (1947) ait été déformée pour n'en retenir que l'idée de grains sonores courts - "de courte durée ", dit Xenakis (1963:61). Dans un système logiciel spécialement conçu pour la synthèse de microsons, Roads (1978: 61) en fixe arbitrairement la durée à 20 millisecondes. On a ainsi abandonné l'image théorique complète, pour n'en retenir que le seul extrême de l'événement sonore élémentaire de durée liminale. Avec une telle durée fixe, on se heurte de surcroît à la difficulté que la largeur de bande perçue ne sera pas la même dans toute l'étendue du registre : elle sera inversement proportionnelle à la fréquence de la sinusoïde émise (cf. Winckel $1967: 53$ ).

42. La composition graphique libre des ondes de pression - c'est-à-dire aussi bien par le dessin proprement dit que par l'application de règles quelconques - est possible dans n'importe quel système, en théorie, puisqu'il s'agit simplement par là de préparer les tables de fonctions de temps qui seront échantillonnées pour former des événements sonores. En pratique, cependant, cette méthode n'est jamais utilisée dans le contexte de systèmes basés primordialement sur des modèles acoustiques prégnants et commandés par des données alphanumériques (MUSIC V,MUSIC10, etc.).Le sys tème UPIC-Unité Polyagogique Informatique du CEMAMu (cf. Xenakis 1976 : 200-02) —, par son orientation vers des données essentiellement graphiques, favorise par contre très nettement cette approche dessinée - d'ailleurs aussi bien de la micro-composition des ondes de pression que de leur utilisation macroscopique. Il est intéressant de noter que, dans la technologie différente des pistes sonores photo-électriques, le cinéaste Norman McLaren (cf. Collins 1976 : 25 et 73-74) utilisa dès 1936 de tels procédés de synthèse sonore. Cependant le système UPIC, ou par exemple les riches possibilités graphiques du SSP (Buxton, et al. 1979), se situent tout de même dans l'optique générale de la mise en situations musicales d'éléments acoustiques autonomes ; il n'y a fondamentalement qu'une différence d'interface, de mode de communication entre le compositeur et l'ordinateur : le symbolisme graphique, plus immédiat mais relativement moins précis, se substitue aux données alphanumériques, indirectes, rébarbatives, mais précises à souhait.

43. Impulsions ("impulses") est pris ici dans le sens d'échantillons.

44. "The changes that best define and illuminate a musical composition are found neither at the lowest nor the highest levels of the hierarchy, but somewhere in between. As the music evolves, we consciously perceive neither individual impusles, which move too fast, nor the overall form of the composition, which moves too slowly. The music is communicated in conscious-time, where we are aware of our awareness of change ".

45. Stuart Smith (1973) fait justement remarquer à ce propos qu'une telle exploitation intensive de variables aléatoires pose de façon critique le problème difficile de la synthèse de phénomènes pseudo-aléatoires (cf. Lorrain 1980).

46. Les premières pages de l'article de Berg, et al. (1980:25-26) sont explicites là-dessus : Koenig a bien conçu son projet comme une extension de la démarche compositionnelle qu'il poursuivait depuis la mise au point de ses systèmes d'algorithmes Project 1 (Koenig 1970a) et Project Two (Koenig 1970b et 
s.d.), dérivés grosso modo de techniques sérielles - combinaisons et permutations de séries de valeurs données ou engendrées aléatoirement - avec adjonction de contrôles stochastiques (tendency masks).

47. L'article de Berg, et al. (1980:26) possède aussi le mérite de ne pas cacher la grande difficulté de la composition directe : "Comment établit-on une relation entre les données et les résultats dans cette approche ... " [ " How does one relate input and output with this approach ... " ] Par le recours à l'analyse de Fourier des ondes sonores obtenues (ibid.: 31-34), il est possible de consolider les expériences intuitives (cf. aussi note 32 ci-dessus).

48. «By substituting the addition of periods for the modulation of frequencies, the composer is able to extend the time and duration control he applies to the macro-events of his composition also to the infrastructure of the event-forming sounds. Thus pitch becomes a result of composition instead of functioning as an element. The same holds true for the concept of sound timbre" ". Il nous a semblé utile de réajuster quelque peu ce passage dans no tre traduction, afin de compenser certaines ambiguïtés et d'éviter de faire allusion à l'expression frequency modulation, qui a pris une signification exclusive depuis l'article de Chowning (1973).

49. Cette idée présente l'avantage de rendre la synthèse sonore en temps réel accessible à pratiquement tout ordinateur. En effet, si une machine peut être globalement trop lente pour des calculs élaborés, ou posséder trop peude mémoire périphérique pour accumuler les échantillons avant leur conversion, elle sera toujours dotée cependant de registres suffisamment rapides.

50. Les algorithmes peuvent être conçus avec un objectif sonore précis, mais leur fonction acoustique ne sera pas nécessairement directement signifiée par leur rédaction objective. Il est évident, bien entendu, que l'expérience permet de prévoir dans une certaine mesure l'effet sonore de divers types d'algorithmes. D'autre part, il est possible, dans ce cadre aussi, de procéder à des élaborations hiérarchiques, et de concevoir des systèmes de synthèse automatique d'algorithmes, au moyen de règles grammaticales par exemple (Holtzman 1979).

51. On peut citer un autre exemple très original de composition directe: le Terrain Reader de Rich Gold (Bischoff, et al. 1978: 25-26). Un " voyageur " se déplace sur un territoire de relief accidenté ; son altitude $z$ est fonction de sa longitude $x$ et de sa latitude $y: z=f(x, y)$; un programme contrôle l'évolution constante de $x$ et de $y$, tandis que $z$ est acheminé vers un convertisseur numériqueanalogique.

52. "Explore the idiomatic capabilities of the computer in the realm of sound synthesis ... systems where the computer is essential for a reason other than the magnitude of the task. Where it could contribute to production of new sorts of sounds. Or processes for producing sound. Or at least new representations of sound".

53. "To hear that which without the computer could not be heard ; to think that which without the computer would not be thought ; to learn that which without the computer would not be learned".

54. Il ne faut pas voir là l'espoir d'une hégémonie musicale de l'ordinateur et du haut-parleur, mais simplement celui de la perfection d'un outil musical souple et maîtrisé, qui trouvera facilement place parmi le concert de ceux que nous possédons déjà. Du reste, l'homme ne peut pas s'empêcher, sinon de progresser, du moins de poursuivre son chemin.

55. "Computer sound synthesis is a medium for obtaining what you specify ; you get all that and only that. At least, one is always in the position of knowing what one has, whether or not that was what one wanted". 


\section{RÉFÉRENCES}

ALLOUIS, J.-F.

1976: "SYTER : un SYstème multiprocesseur de synthèse digitale TEmps Réel », Cahier recherche/musique, $\mathrm{N}^{\circ} 3$, «Synthétiseur/ ordinateur ", 62-90.

1979: "Use of High-Speed Microprocessors for Digital Synthesis », Computer Music Journal, III/1, 14-16.

ARFIB, D.

1978: Digital Synthesis of Complex Spectra by means of Multiplication of Non-Linear Distorted Sine Waves. New York : Audio Engineering Society (preprint $n^{\circ} 1319(C-2)$ ).

BATTIER, M.

1980: Le langage de synthèse MUSIC10. Paris: IRCAM (document interne).

BERG, P.

1975: ASP - Automated Synthesis Program. Utrecht : Instituut voor Sonologie.

1979: «PILE - A Language for Sound Synthesis », Computer Music Journal, III/1, 30-37.

BERG, P., ROWE, R., and THERIAULT, D.

1980: "SSP and Sound Description», Computer Music Journal, IV/1, 25-35.

BISCHOFF, J., GOLD, R., and HORTON, J.

1978: "Music for an Interactive Network of Microcomputers », Computer Music Journal, II/3, 24-29.

BLUM, T.

1979: « Herbert Brün : Projected Sawdust », Computer Musical Journal, III/1, 6-7.

BOWLES, E.A.

1970: "Musicke's Handmaiden: Or Technology in the Service of the Arts ", in Lincoln, H.B., éd., The Computer and Music, Ithaca, N.Y.: Cornell University Press, 3-20.

BRACEWELL, $R$.

1965: The Fourier Transform and Its Applications. New York: McGraw-Hill.

BUXTON, W.

1978: Design Issues in the Foundation of a Computer-Based Tool for Music Composition. Toronto: University of Toronto, Computer Systems Research Group (Technical Report GSRC-97).

BUXTON, W., SNIDERMAN, R., REEVES, W., PATEL, S., and BAECKER, $R$.

1979: "The Evolution of the SSSP Score Editing Tools », Computer Music Journal, III/4, 14-25. 
BUXTON, W., REEVES, W., FEDORKOW, G., SMITH, K.C., and BAECKER, $R$.

1980: "A Microcomputer-Based Conducting System », Computer Music Journal, IV/1, 8-21.

CANN, R.

1979a: «An Analysis/Synthesis Tutorial (Part I) », Computer Music Journal, III/3, 6-11.

1979b: "An Analysis/Synthesis Tutorial (Part II », Computer Music Journal, III/4, 9-13.

1980: "An Analysis/Synthesis Tutorial (Part III)», Computer Music Journal, IV/1, 36-42.

CHADABE, J.

1977: "Some Reflexions on the Nature of the Landscape within which Computer Music Systems are Designed ", Computer Music Journal, I/3, 5-11.

CHADABE, J. and MEYERS, $R$.

1978: "An Introduction to the PLAY Program », Computer Music Journal, II/1, 12-18.

CHARLES, D.

1978: Gloses sur John Cage. Paris: Union générale d'éditions (coll. $\left.10-18 n^{\circ} 1212\right)$.

CHION, M.

1976: «Éditorial », Cahier recherche/musique, $\mathrm{N}^{\circ} 3$, «Synthétiseur/ ordinateur $"$, 3-4.

CHOWNING, J.

1973: "The Synthesis of Complex Audio Spectra by Means of Frequency Modulation ", Journal of the Audio Engineering Society, $\mathrm{XXI} / 7,526-34$.

COLLINS, $\mathrm{M}$.

1976: Norma McLaren. Ottawa: Canadian Film Institute.

CULLMANN, G., DENIS-PAPPIN, M., et KAUFMANN, A.

1970: Cours de calcul informationnel appliqué. Paris: Albin Michel (coll. Progrès des sciences et techniques).

ECO, U.

1965: L'oeuvre ouverte. Paris: Éditions du Seuil.

GABOR, D.

1947: "Acoustical Quanta and the Theory of Hearing ", Nature, CLIX/4044, 591-94.

GOUDOT, A.

1952: Les quanta et la vie. Paris: P.U.F. (coll Que sais-je? $N^{\circ} 530$ ). HAYNES, $S$.

1980: "The Musician-Machine Interface in Digital Sound Synthesis ", Computer Music Journal, IV/4, 23-44. 
HILLER, L.A., Jr.

1970: "Music Composed with Computers - A Historical Survey ", in Lincoln, H.B., éd., The Computer and Music. Ithaca, N.Y.: Cornell University Press, 42-96.

HOLTZMAN, S.R.

1979: "An Automated Digital Sound Synthesis Instrument 》, HOWE, H.S. Computer Music Journal, III/2, 53-61.

1975: Electronic Music Synthesis : Concepts Facilities, Techniques. New York: W.W. Norton.

KAEGI, W.

1971: "Musique et technologie dans l'Europe de 1970 », Musique et technologie, Paris: Richard-Masse, 9-30 (La revue musicale, $\mathrm{N}^{\circ}$ 268-269).

KOENIG, G.M.

1970a: «Project 1 », Electronic Music Reports, $N^{\circ} 2$, Utrecht: Instituut voor Sonologie.

1970b: "Project Two », Electronic Music Reports, N 3, Utrecht: Instituut voor Sonologie.

s.d.: Computer Composition. Utrecht: Instituut voor Sonologie.

LALOY, L.

1909: Rameau. Paris: Alcan (coll. Les maîtres de la musique).

LASKE, $\mathrm{O}$.

1978: "Considering Human Memory in Designing User Interfaces for

Computer Music », Computer Music Journal, II/4, 39-49.

LORRAIN, D.

1980: "A Panoply of Stochastic 'Cannons' », Computer Music Journal, IV/1, 53-81.

MATHEWS, M.V.

1969: The Technology of Computer Music. Cambridge, Mass.: MIT Press.

MATHEWS, M.V., and MOORE, F.R.

1970: "GROOVE - A Program to Compose, Store and Edit Functions of Time", Communications of the ACM (Association for Computing Machinery), 13-12, pp. 715-21.

MOLES, A.

1972: Théorie de l'information et perception esthétique. Paris: MOORE, F.R. Denoël (coll. Grand format Médiations).

1978a: «An Introduction to the Mathematics of Digital Signal Processing (Part I) », Computer Music Journal, II/1, 38-47.

1978b: « An Introduction to the Mathematics of Digital Signal Processing (Part II) », Computer Music Journal, II/2, 38-60. 
MOORER, J.A.

1977: "Signal Processing Aspects of Computer Music », Computer Music Journal, I/1, 4-37.

1978: "How Does a Computer Make Music? », Computer Music Journal, II/1, 32-37.

MOORER, J.A., CHAUVEAU, A., ABBOTT, C., EASTY, P., and LAWSON, J.

1979: "The 4C Machine », Computer Music Journal, III/3, 16-24.

NATTIEZ, J.-J.

1975: Fondements d'une sémiologie de la musique. Paris: Union générale d'éditions (coll. 10-18, $\mathrm{N}^{\circ} 1017$ ).

PASQUET, Y.-M.

1979: Pour une analyse de la pièce IV du Marteau sans Maître de Pierre Boulez. Paris: IRCAM (document non publié).

PIMONOV, L.

1962: Vibrations et régime transitoire. Paris; Dunod (Coll. technique et scientifique du C.N.E.T.).

REVAULT D'ALLONNES, $\mathrm{O}$.

1973: La création artistique et les promesses de la liberté. Paris: Klincksieck.

RICHER, J.-L.

1979: La synthèse des sons par ordinateur. Manuel de référence MUSIC V. Paris: IRCAM (document interne).

RIOTTE, A.

1974: "Informatique musicale : junction nouvelle entre art et science », Eurospectra, XIII/1, 3-15.

RISSET, J.-C.

1971: "Quelques remarques sur les musiques pour ordinateur et l'interprétation ", Musique en jeu, $\mathrm{N}^{\circ} 3,5-11$.

ROADS, Curtis

1978: "Automated Granular Synthesis of Sound ", Computer Music Journal, II/2, 61-62.

1979a: " A Tutorial on Non-Linear Distortion or Waveshaping Synthesis », Computer Music Journal, III/2, 29-34.

1979b: " An Interview with Harold Cohen », Computer Music Journal, III/4, 50-57.

ROZENBERG, $M$.

1979: " Microcomputer-Controlled Sound Processing Using Walsh Functions », Computer Music Journal, III/1, 42-47.

SCHAEFFER, $P$.

1977: Traité des objets musicaux. Paris: Éditions du Seuil (coll. Pierres vives).

SMITH, I.S.

1973: "Communications », Perspectives of New Music, XI/2, 269-77. STEIGLITZ, K.

1974: An Introduction to Discrete Systems, New York: John Wiley. 
TRUAX, B.

1977: "The POD System of Interactive Composition Programs ", Computer Music Journal, I/3, 30-39.

VERCOE, $\mathrm{B}$.

1971: "The Music 360 Language for Sound Synthesis ", American Society of University Composers Proceedings, $\mathrm{N}^{\circ} 6,16-21$.

WALLRAFF, D.

1979: "The DMX-1000 Signal processing Computer », Computer Music Journal, III/4, 44-49.

WINCKEL, F.

1967: Music, Sound and Sensation, New York: Dover. XENAKIS, I.

1963: Musiques formelles. Paris: Richard-Masse (La revue musicale, $\mathrm{N}^{\circ} 253-254$ ).

1971: Formalized Music. Bloomington, Ind.: Indiana University Press.

1976: Musique. Architecture. Tournai; Casterman (coll. Synthèses contemporaines).

1978: Séminaire à l'Université de Paris I, automne. 